\title{
Resistin Levels in Perivascular Adipose Tissue and Mid-Term Mortality in Patients Undergoing Coronary Artery Bypass Granting
}

\author{
Maciej RACHWALIK ${ }^{1}$, Marta OBREMSKA ${ }^{2}$, Dorota ZYŚKO ${ }^{3}$, Małgorzata \\ MATUSIEWICZ ${ }^{4}$, Marcin PROTASIEWICZ ${ }^{5}$, Marek JASIŃSKI ${ }^{6,7}$
}

${ }^{1}$ Department of Cardiac Surgery, Wroclaw Medical University, Wroclaw, Poland, ${ }^{2}$ Department of Preclinical Research, Wroclaw Medical University, Wroclaw, Poland, ${ }^{3}$ Department of Emergency Medicine, Wroclaw Medical University, Wroclaw, Poland, ${ }^{4}$ Department of Medical Biochemistry, Wroclaw Medical University, Wroclaw, Poland, ${ }^{5}$ Department of Cardiology, Wroclaw Medical University, Wroclaw, Poland, ${ }^{6}$ Department of Cardiac Surgery, Wroclaw Medical University, Wroclaw, Poland, ${ }^{7}$ Children's Memorial Pediatric Health Institute, Warsaw, Poland

Received February 15, 2021

Accepted May 4, 2021

Epub Ahead of Print June 2, 2021

\section{Summary}

Higher serum resistin levels were reported to be associated with increased mortality risk. We aimed to assess the predictive value of resistin levels in perivascular adipose tissue (PVAT) around the left main coronary artery (LMCA) for mid-term survival of patients with advanced coronary artery disease (CAD). This was a prospective study including patients referred for elective coronary artery grafting in 2016 and 2017, performed using a standard approach. A sample of PVAT was harvested and resistin levels were measured using an enzyme-linked immunosorbent assay. Patients were followed from the day of the procedure until March 2021. In each patient, the SYNTAX score and EuroSCORE II were calculated. The study included 108 patients aged $68.1 \pm 7.9$ years, including 83 men $(76.9 \%)$. The duration of follow-up was 731 (range, 275-1020) for nonsurvivors and 1418 median (range, 1174-1559) for survivors $(p<0.001)$. Patients who died had a higher SYNTAX score, higher EuroSCORE II, and lower resistin levels in PVAT than survivors ( $p<0.001, p=0.004$, and $p=0.041$, respectively). A stepwise regression analysis revealed that survival was related to resistin concentrations above the median value (hazard ratio [HR], 4.67, $95 \% \mathrm{CI}, 1.02-21.4, p=0.048)$ and EuroSCORE II (used as continuous variable, $\mathrm{HR}, 1.55,95 \% \mathrm{CI}, 1.16-2.07$, $p=0.003)$. The mid-term mortality in patients with advanced CAD is associated with low resistin concentrations in PVAT surrounding the LMCA.

\section{Key words}

Resistin • CABG • Adipose tissue

\author{
Corresponding author \\ Maciej Rachwalik, Department of Cardiac Surgery \\ ul. Borowska 213 50-556 Wrocław Poland. E-mail: \\ maciej.rachwalik@umed.wroc.pl
}

\section{Introduction}

Numerous studies have shown the role of local inflammation in the development of atherosclerotic plaque (Nosalski et al. 2017). Perivascular adipose tissue (PVAT), which surrounds most of the vasculature, has been implicated in the pathogenesis of atherosclerosis. Its proximity to the arterial wall creates conditions for their mutual interaction. The tissue exerts both proinflammatory and anti-inflammatory properties. A balance between these opposite effects plays an important role in regulating vascular function both in physiological and pathological processes.

One of the adipokines secreted by PVAT is resistin. It acts as a proinflammatory factor but its exact physiological significance is unknown. The serum resistin concentration positively correlates with the presence and severity of coronary atherosclerosis (Krecki et al. 2008, Laframboise et al. 2012). Moreover, in a meta-analysis of patients with type 2 diabetes mellitus and coronary artery disease (CAD), higher circulating serum resistin levels were associated with an increased 
risk of mortality (Fontane et al. 2015). Circulating resistin is mainly derived from macrophages and monocytes and, to a lesser extent, from adipocytes. The secretory role of monocytes has been confirmed by the observation of simultaneous increase of resistin and monocyte chemoattractant protein-1 (MCP-1) generated by these cells in the event of an induced inflammatory reaction (Saracevic et al. 2020). Therefore, the concentrations of resistin in serum may not reflect those in PVAT, while resistin concentrations in PVAT alone may differ depending on the coronary artery.

The aim of this study was to assess the predictive value of resistin concentrations in PVAT surrounding the left main coronary artery (LMCA) for mid-term survival of patients with advanced CAD.

\section{Methods}

This was a prospective study comprising patients referred for elective coronary artery bypass grafting (CABG)-on pump method due to advanced CAD. Inclusion criteria for the study were: three-vessel coronary artery disease (3VD), age less than 80 years, written consent to participate in the study. The exclusion criteria were concomitant severe or moderate valvular disease requiring an additional procedure, and left ventricular ejection fraction (LVEF) lower than $30 \%$.

We enrolled 108 consecutive patients who met the inclusion criteria and underwent CABG in 2016 and 2017. We excluded patients who died up to 120 days after surgery from the final analysis, $(n=3)$, which may be related to post-CABG perioperative complications. Demographic and clinical data (history of arterial hypertension, stroke, myocardial infarction, diabetes, and atrial fibrillation) were collected. Before $\mathrm{CABG}$, each patient underwent echocardiography and the measurement of LVEF. Angiography results of each participant were analyzed by an interventional cardiologist. The anatomic-based SYNTAX score (Synergy Between Percutaneous Coronary Intervention With Taxus and Cardiac Surgery) and the degree of left main stenosis were assessed using the SYNTAX Score Calculator by a trained analyst blinded to patient characteristics. EuroSCORE II (European System for Cardiac Operative Risk Evaluation) was calculated for each patient as part of routine clinical practice. The life status of patients was determined on the basis of data obtained from the Ministry of Digital Affairs for March 18, 2021. The follow-up lasted from the date of the cardiac surgery to March 18, 2021, or until patient's death.

The CABG procedure was performed using a standard sternotomy approach. Cardiopulmonary bypass was established with an aortic cannula inserted to the ascending aorta and a venous return through the cannula to the right atrial appendage. After the crossclamp of the aorta and administration of antegrade cold blood cardioplegia (according to the del Nido protocol), the heart was lifted and a sample of PVAT around the LMCA was harvested using the surgical scissors and blade. A small portion of the sample $(3 \times 3 \times 3 \mathrm{~mm})$ was immediately frozen at a temperature of $-80^{\circ} \mathrm{C}$ and stored until further analysis. After sampling, the bypasses were performed using a standard approach with the internal thoracic arteries and saphenous veins.

For the homogenization of tissue samples, a FastPrep homogenizer (MP Biomedicals, Santa Ana, CA, USA) and PMSF buffer were used. Centrifugation was performed twice for $10 \mathrm{~min}$ at $14000 \mathrm{x} \mathrm{g}$ and a temperature of $4{ }^{\circ} \mathrm{C}$. The second centrifugation was performed just before the analysis. The concentration of resistin in PVAT was measured using Human Resistin Quantikine ELISA Kit (R\&D Systems, Minneapolis, MN, USA) according to the manufacturer's instructions and expressed in $\mathrm{ng} / \mathrm{g}$ of tissue. If the laboratory result was marked as exceeding the maximum, the maximum value obtained in other measurements was used for analysis.

The study was approved by the local ethics committee (KB 392/2016).

\section{Statistical analysis}

Continuous variables were presented as mean and standard deviation or median and interquartile range (IQR) according to their distribution and compared with the $t$ test or the Mann-Whitney test, respectively. Categorical variables were presented as numbers and percentages and compared with the $\chi^{2}$ test. Patients were divided into those who survived and those who died during follow-up. The continuous variables that differed between groups in a univariate analysis with a $p$ value of at least less than 0.15 were included in stepwise multivariate analyses as continuous or dichotomized variables according to their median value or the upper limit of normal when it was known for a given variable. The Kaplan-Meier curves were generated to visualize differences in survival between groups classified on the basis of the median values of parameters that differed in 
the univariate analysis between survivors and nonsurvivors. In the case of continuous variables, the cutoff points were selected on the basis of their median values and the subgroups with the values lower and higher than the median were compared. The stepwise Cox proportional hazards regression analysis was performed to assess the associations between survival and independent variables. A $p$ value of less than 0.05 was considered significant.

Table 1. Demographic and clinical characteristics of survivors and nonsurvivors patients

\begin{tabular}{|c|c|c|c|}
\hline Parameter & $\begin{array}{l}\text { Survivors } \\
(\mathrm{n}=91)\end{array}$ & $\begin{array}{l}\text { Nonsurvivors } \\
\quad(n=14)\end{array}$ & $p$ value \\
\hline Age, years, mean $\pm S D$ & $67.7 \pm 8$ & $70.5 \pm 8.0$ & 0.239 \\
\hline Male sex, $n(\%)$ & $71(78)$ & $10(71)$ & 0.584 \\
\hline$B M I, \mathrm{~kg} / \mathrm{m}^{2}$, mean $\pm S D$ & $27.5 \pm 4.4$ & $28.9 \pm 6.7$ & 0.314 \\
\hline Diabetes, $n(\%)$ & $33(36)$ & $8(57)$ & 0.136 \\
\hline Hypertension, $n(\%)$ & $80(88)$ & $13(93)$ & 0.588 \\
\hline Previous infarction, $n(\%)$ & $50(55)$ & $9(64)$ & 0.511 \\
\hline Previous $C A B G / P C I, n(\%)$ & $20(22)$ & $5(36)$ & 0.432 \\
\hline Previous stroke, $n(\%)$ & $8(9)$ & $1(7)$ & 0.758 \\
\hline History of atrial fibrillation, $n(\%)$ & $2(2)$ & $3(21.0)$ & 0.013 \\
\hline Renal insufficiency, $n(\%)$ & $4(4)$ & $0(0)$ & 0.96 \\
\hline Left ventricular ejection fraction, $\%$ & $55.5 \pm 8.1$ & $53.6 \pm 9.6$ & 0.445 \\
\hline Left ventricular diastolic dimension, $n(\mathrm{~mm})$ & $52.9 \pm 4.4$ & $49.8 \pm 5.24$ & 0.05 \\
\hline$I V S, n(m m)$ & $12.9 \pm 1.7$ & $12.1 \pm 1.2$ & 0.18 \\
\hline$P W d, n(m m)$ & $10.6 \pm 1.7$ & $10.9 \pm 1.7$ & 0.49 \\
\hline Left ventricular mass, $n$ (mg) & $255.8 \pm 51.1$ & $226.4 \pm 54.5$ & 0.085 \\
\hline$S Y N T A X$ score, mean $\pm S D$ & $35.3 \pm 11.7$ & $52.4 \pm 14.4$ & $<0.001$ \\
\hline Median SYNTAX score, 34.95 (28.75-45.00), n (\%) & $40(44)$ & $13(93)$ & 0.014 \\
\hline EuroSCORE II, median (IQR) & $1.18(0.84-1.69)$ & $1.44(1.23-2.59)$ & 0.061 \\
\hline Median EuroSCORE II >1.24, n (\%) & $41(45)$ & $10(71)$ & 0.066 \\
\hline LMCA stenosis $\geq 50 \%, n(\%)$ & $40(44)$ & $8(57)$ & 0.357 \\
\hline LMCA stenosis, median (IQR) & $40(0-60)$ & $50(30-80)$ & 0.285 \\
\hline Resistin in the PVAT surrounding LMCA, ng/g, median (IQR) & $124.9(32.7-362.7)$ & $61(6.3-104.6)$ & 0.094 \\
\hline Resistin in the PVAT surrounding LMCA LM > median, $n(\%)$ & $40(44)$ & $11(79)$ & 0.016 \\
\hline Glycemia, $m g \%$, median (IQR) & $119 \pm 35$ & $127 \pm 47$ & 0.016 \\
\hline Creatinine, $m g \%$, mean $\pm S D$ & $1.12 \pm 0.36$ & $1.06 \pm 0.21$ & 0.55 \\
\hline$e G F R, \mathrm{ml} / \mathrm{min} / 1.73 \mathrm{~m}^{2}$, mean $\pm S D$ & $71.7 \pm 20.5$ & $70.1 \pm 13.1$ & 0.786 \\
\hline Hemoglobin, $g / l$, mean $\pm S D$ & $13.9 \pm 1.5$ & $13.5 \pm 1.8$ & 0.379 \\
\hline
\end{tabular}

BMI - body mass index, CABG - coronary artery bypass graft, eGFR - estimated glomerular filtration rate, LMCA - left main coronary artery, PCI - percutaneous coronary intervention, PVAT - perivascular adipose tissue, IVS - intraventricular septum, PWd - Posterior wall diameter.

\section{Results}

The study group included 108 patients aged $68.1 \pm 7.9$ years, including 83 men $(76.9 \%)$. All patients underwent a successful CABG due to advanced CAD. Based on the information obtained from the Ministry of Digital Affairs, we excluded 3 patients who died in the early follow-up period - up to 120 days after the procedure. On this basis, we found that 14 patients died 120 days after the surgery.103 patients were included for further analysis. The duration of follow-up was 731 (range, 275-1020) for nonsurvivors and 1418 median (range, 1174-1559) for survivors $(p<0.001)$. Therefore, mean follow-up for patients who did not die early after surgery was IQR 1407 (1152-1552) range 122-1694. The median follow-up duration for the whole group was 
IQR 1405.5 (1142-1552) range 26-1694. The assessment of mortality in the study group refers to March 18, 2021.

The comparison of clinical and laboratory parameters between nonsurvivors and survivors is presented in Table 1. There were no differences in the history of diabetes, hypertension, infarction stroke, and revascularization of the coronary arteries between groups. History of atrial fibrillation was more common in nonsurvivors. Laboratory parameters were similar between groups, except a trend for higher glucose levels in patients who died.

The severity of CAD measured by the SYNTAX score was significantly higher in nonsurvivors. The median SYNTAX score in the study group was 34.95 (IQR, 28.75-45.00), and the SYNTAX score above the median value was more frequent in nonsurvivors. Similarly, EuroSCORE II was higher in nonsurvivors than in survivors. The median EuroSCORE II for all patients was 1.24 (IQR, 0.87-1.84).

The median resistin concentration in PVAT for the whole study group was $106.1 \mathrm{ng} / \mathrm{g}$ (IQR, 31.1-358.2). The concentrations were significantly higher in survivors (Table 1). Patients with a EuroSCORE II below the median value had higher resistin concentrations than those with the score above the median (Fig. 1). Among patients with the SYNTAX score below the median value, the median resistin concentration was $53.1 \mathrm{ng} / \mathrm{g}$ (IQR, 21.5-134.4), as compared with $54.9 \mathrm{ng} / \mathrm{g}$ (IQR, 16.5-377.0) in those with the score above the median $(p=0.49)$.

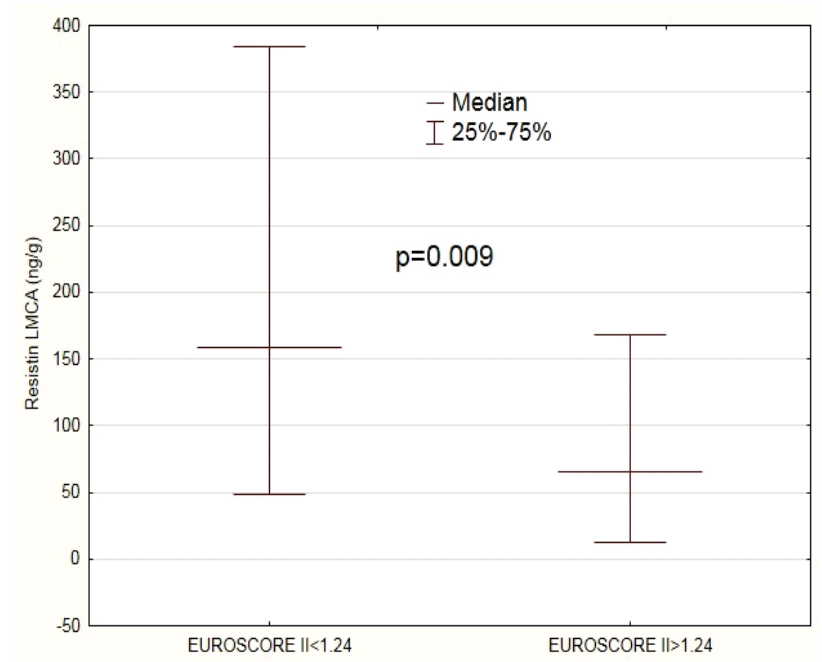

Fig. 1. Resistin concentrations in the perivascular adipose tissue surrounding the left main coronary artery in patients with EuroSCORE II below and above the median value
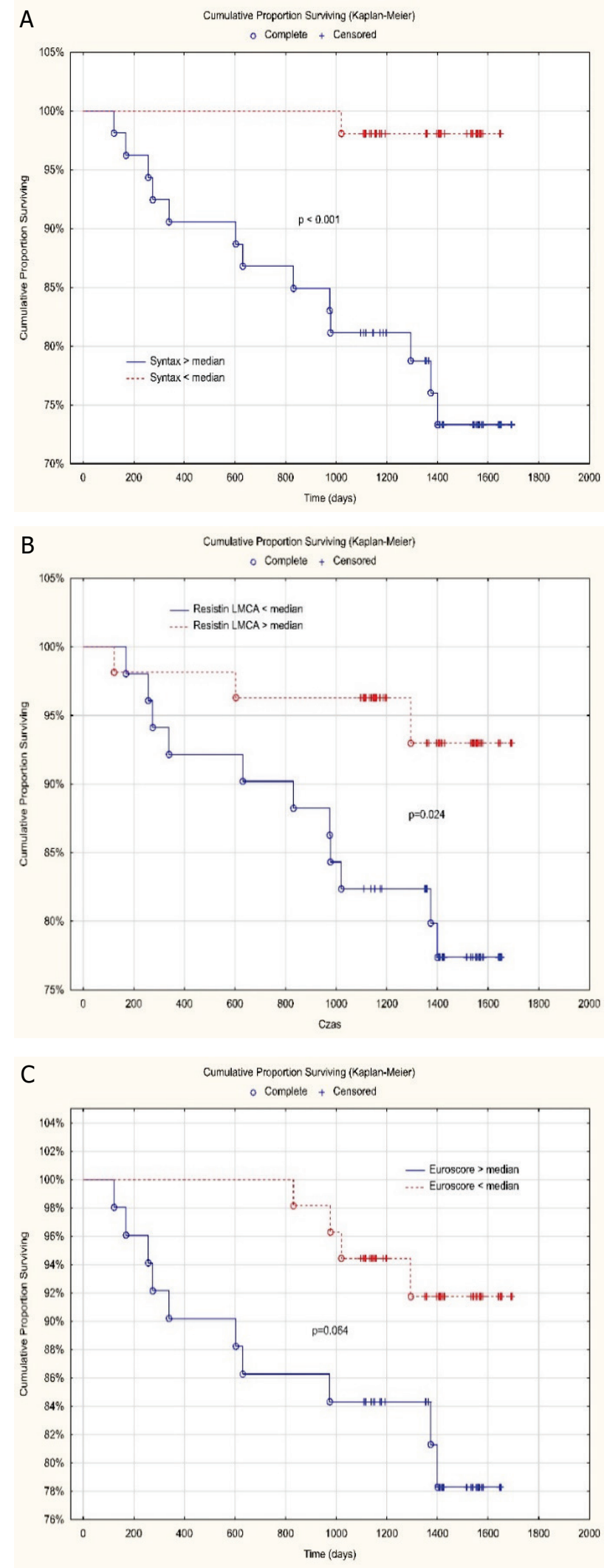

Fig. 2. The Kaplan-Meier survival curves for patients who underwent coronary artery bypass grafting divided according to the median value of: A - SYNTAX score (median, 34.95);

B - resistin levels in the perivascular adipose tissue surrounding the left main coronary artery (median, $106.1 \mathrm{ng} / \mathrm{g}$ ); C - EuroSCORE II (median, 1.24) 
The stepwise Cox proportional hazards regression analysis revealed that survival was related to the resistin concentration above the median value (hazard ratio $[\mathrm{HR}], 4.67,95 \% \mathrm{CI}, 1.02-21.4, p=0.048)$ and EuroSCORE II (used as a continuous variable, HR, 1.55, $95 \%$ CI 1.16-2.07, $p=0.003$ ). The Kaplan-Meier survival curves for the subgroups of patients divided according to the median values of resistin, EuroSCORE II, and SYNTAX score are presented in Fig. 2.

\section{Discussion}

The main finding of our study is that the midterm survival of patients after CABG is associated with a higher concentration of PVAT resistin surrounding the LMCA. Additionally, the factor that favored survival in our study was a lower EuroSCORE II index, which was demonstrated in large trials. EuroSCORE II is considered the standard scoring system for the assessment of perioperative mortality risk in cardiac surgery procedures in routine clinical practice (Nashef et al.2016, Sulivan et al. 2016). However, the scale can be also used to assess the risk of mid-term mortality in patients undergoing combined aortic valve replacement and CABG (Spoliopoulos et al. 2014). Therefore, our finding on the relationship between a higher EuroSCORE II value and mortality is concordant with previous reports. The novelty of our study is the observed association between the higher resistin level in PVAT surrounding the LMCA and better mid-term prognosis in patients undergoing CABG.

The relationship between serum resistin levels and mortality has not been fully elucidated. Moreover, the serum levels do not reflect those in PVAT. In the LURIC study (Ludwigshafen Risk and Cardiovascular Health), which assessed patients with angiographically confirmed coronary atherosclerosis, no relationship between serum resistin levels and coronary atherosclerosis or cardiovascular mortality was reported (Pilz et al. 2007). However, the study found a positive correlation between resistin and inflammatory markers. Moreover, elevated resistin levels were reported as an indicator of heart muscle injury and were detected in patients with acute coronary syndromes (Lubos et al. 2007, Wang et al. 2009).

Atherosclerosis is caused by lipids accumulation in early stages and thereafter the inflammation of the coronary arteries which consumes PVAT and leads to its local cachexia (Antoniades et al. 2019). A study on patients with chronic inflammation showed a decrease in the amount of adipose tissue and its antiatherosclerotic properties in individuals with and without significant cardiovascular disease (Antonopoulos et al. 2014). Similarly, in patients with heart failure, cachexia was shown to be related to higher mortality (Carbone et al. 2013). Furthermore, noninvasive studies assessing PVAT indicated that not only the amount but also the properties of adipose tissue are important for patient outcomes. The presence of a higher amount of PVAT was related to the occurrence of CAD in the general population and lowrisk patients (Bos et al. 2015, Dey et al. 2012, Ito et al. 2013, Versteylen et al. 2012). However, in the SMART study (Secondary Manifestations of ARTerial Disease), Franssens et al showed that, in addition to the volume, also the quality of epicardial adipose tissue is associated with CAD in high-risk patients (Franssens et al. 2017). They suggested that the radiodensity of epicardial adipose tissue is correlated with coronary atherosclerosis. A potential explanation for our results may be the progressive cachexia of PVAT, expressed as a decrease in resistin concentrations in epicardial fat surrounding LMCA in the 3VD group. PVAT cachexia expressed in this way was statistically higher in the nonsurvivor group.

Recently, a new biomarker has been developed for the assessment of inflammation in PVAT, namely, the fat attenuation index (FAI) (Antonopoulos et al. 2014). Local inflammation leads to a decrease in the size, lipid accumulation, and differentiation of adipocytes, which correlates with a gradient in attenuation of pericoronary or epicardial fat from more negative to less negative Hounsfield unit values on computed tomography angiography, which is reflected by an increase in the FAI (Mancio et al. 2018). In the CRIPS-CT study, a J-shape association between the FAI of the PVAT and the risk of cardiac death was shown (Oikonomou et al. 2018). Patients with a very high FAI had approximately 9-fold higher risk of cardiac death. Additionally, in this study, the FAI was weakly correlated with plasma C-reactive protein, which can be multifactorial in origin and come from other sources.

Our patients had advanced atherosclerosis and it could be presumed that there were local depletion of reserves on resistin in PVAT in patients with poor outcome. Moreover, nonsurvivors also had a higher EuroSCORE II despite the fact that all patients underwent elective $\mathrm{CABG}$ and had preserved LVEF. The significance of EuroSCORE II for predicting mid-term outcome lies in its components, namely, older age, male 
sex, the presence of noncardiac atherosclerosis, previous myocardial infarction, $\mathrm{CABG}$, and stroke.

Due to the effect of PVAT on the vasculature, the assessment of local resistin concentrations may be relevant for the understanding of atherosclerosis development, which may have important implications for the management and prognosis of patients with CAD.

In the face of studies indicating that proinflammatory cytokines produced by adipose tissue can induce myocardial dysfunction and promote the formation of atherosclerotic plaques, our results can appear paradoxical. However, no studies comparing the levels of resistin in plasma and adipose tissue have been performed to date, and it cannot be assumed that this relationship is proportional, especially that resistin in blood comes from various sources. In addition, the so-called obesity paradox is observed, whereby patients with obesity and heart failure or coronary heart disease show higher survival rates than normal-weight or underweight individuals (Horwich et al. 2018, Elagizi et al. 2018).

The obtained results are also in line with findings of Al-Nimer et al. who found that high serum leptin levels are associated with negative coronary computed tomography (Al-Nimer et al. 2018).

The main limitation of the study is a small sample size. However, the median follow-up was almost 4 years, therefore, the analysis is justified as a mid-term survival. Moreover, we did not assess serum resistin levels. The serum resistin comes from many sources mainly from macrophages and monocytes (Saracevic et al. 2020). Therefore, the measurement in the PVAT may be more specific for assessing the significance of local factors on the outcomes of coronary atherosclerosis. Moreover, no additional characterization of adipose tissue was presented using the measurements of adipose tissue thickness, FAI (Fat Attenuation Index) obtained from others modalities of imaging. Another limitation is that we assessed all-cause mortality and not cardiovascular mortality. All-cause mortality is used as a primary endpoint in numerous clinical trials because it is less sensitive to bias. Moreover, the most common cause of death in this group of patients is cardiovascular death.

Our results cannot be easily compared with previous studies because only serum resistin concentrations are typically measured. The adipose tissue surrounding cardiac muscle could be obtained only during cardiac surgery whereas the blood from peripheral veins is much easier to draw.

\section{Conclusions}

In conclusion, our study showed that mid-term mortality in patients with multivessel CAD is associated with low resistin concentrations in the PVAT surrounding the LMCA. Higher resistin concentrations in this tissue are associated with a lower EuroSCORE II in patients with multivessel CAD.

\section{Conflict of Interest}

There is no conflict of interest.

\section{Acknowledgements}

The study was approved by the local ethics committee [KB 392/2016]. All patients provided written informed consent to participate in the study.

\section{References}

AL-NIMER MS., ALHUSSEINY AH, IBRAHIM AK: Assessment of serum adiponectin and leptin levels in patients with ischaemic heart disease: an association with the ejection fraction, coronary calcium score and coronary Folia Cardiologica 13: 204-209, 2018. https://doi.org/10.5603/FC.2018.0041

ANTONIADES C KOTANIDIS CP, BERMAN DS: State-of-the-art review article. Atherosclerosis affecting fat: what can we learn by imaging perivascular adipose tissue? J Cardiovasc Comput Tomogr 13: 288-296, 2019. https://doi.org/10.1016/j.jcct.2019.03.006

ANTONOPOULOS AS, MARGARITIS M, COUTINHO P, DIGBY J, PATEL R, PSARROS C, NTUSI N, KARAMITSOS TD, LEE R, DE SILVA R, PETROU M, SAYEED R, DEMOSTHENOUS M, BAKOGIANNIS C, WORDSWORTH PB, TOUSOULIS D, NEUBAUER S, CHANNON KM, ANTONIADES C: Reciprocal effects of systemic inflammation and brain natriuretic peptide on adiponectin biosynthesis in adipose tissue of patients with ischemic heart disease. Arterioscler Thromb Vasc Biol 34 : 2151-2159, 2014. https://doi.org/10.1161/ATVBAHA.114.303828 
ANTONOPOULOS AS, SANNA F, SABHARWAL N, THOMAS S, OIKONOMOU EK, HERDMAN L, MARGARITIS M, SHIRODARIA C, KAMPOLI AM, AKOUMIANAKIS I, PETROU M, SAYEED R, KRASOPOULOS G, PSARROS C, CICCONE P, et al. Detecting human coronary inflammation by imaging perivascular fat. Sci Transl Med 9: 2017. https://doi.org/10.1126/scitranslmed.aal2658

BOS D, SHAHZAD R, VAN WALSUM T, VAN VLIET LJ, FRANCO OH, HOFMAN A, NIESSEN WJ, VERNOOIJ MW, VAN DER LUGT A: Epicardial fat volume is related to atherosclerotic calcification in multiple vessel beds. Eur Heart J Cardiovasc Imaging 16: 1264-1269, 2015. https://doi.org/10.1093/ehjci/jev086

CARBONE S, CANADA JM, BILLINGSLEY HE, SIDDIQUI MS, ELAGIZI A, LAVIE CJ: Obesity paradox in cardiovascular disease: where do we stand? Vasc Health Risk Manag 15: 89-100, 2019. https://doi.org/10.1016/j.pcad.2019.08.004

DEY D, NAKAZATO R, LI D, BERMAN DS: Epicardial and thoracic fat: noninvasive measurement and clinical implications. Cardiovasc Diagn Ther 2: 85-93, 2012.

ELAGIZI A, KACHUR S, LAVIE CJ, CARBONE S, PANDEY A, ORTEGA FB, MILANI RV: An overview and update on obesity and the obesity paradox in cardiovascular diseases. Prog Cardiovasc Dis 61: 142-150, 2018. https://doi.org/10.1016/j.pcad.2018.07.003

FONTANA A, SPADARO S, COPETTI M, SPOTO B, SALVEMINI L, PIZZINI P, PIZZINI P, FRITTITTA L, MALLAMACI F, PELLEGRINI F, TRISCHITTA V, MENZAGHI C: Association between resistin levels and all-cause and cardiovascular mortality: a new study and a systematic review and meta-analysis. PLoS One 10: e0120419, 2015. https://doi.org/10.1371/journal.pone.0120419

FRANSSENS BT, NATHOE HM, VISSEREN FL, VAN DER GRAAF Y, LEINER T; SMART Study Group. Relation of epicardial adipose tissue radiodensity to coronary artery calcium on cardiac computed tomography in patients at high risk for cardiovascular disease. Am J Cardiol 119: 1359-1365, 2017. https://doi.org/10.1016/j.amjcard.2017.01.031

HORWICH TB, FONAROW GC, CLARK AL: obesity and obesity paradox in heart failure. Prog Cardiovasc Dis 61: 151-156, 2018. https://doi.org/10.1016/j.pcad.2018.05.005

ITO T, SUZUKI Y, EHARA M, MATSUO H, TERAMOTO T, TERASHIMA M, et al.: Impact of epicardial fat volume on coronary artery disease in symptomatic patients with a zero calcium score. Int J Cardiol 167: 2852-2858, 2013. https://doi.org/10.1016/j.ijcard.2012.07.026

KRECKI R, DROZDZ J, SZCZEŚNIAK P, ORSZULAK-MICHALAK D, KRZEMIŃSKA-PAKUŁA M: Novel atherogenesis markers for identification of patients with a multivessel coronary artery disease. Kardiol Pol 66: 1173-1180, 2008.

LAFRAMBOISE WA, DHIR R, KELLY LA, PETROSKO P, KRILL-BURGER JM, SCIULLI CM, LYONS-WEILER MA, CHANDRAN UR, LOMAKIN A, MASTERSON RV, MARROQUIN OC, MULUKUTLA SR, MCNAMARA DM: Serum protein profiles predict coronary artery disease in symptomatic patients referred for coronary angiography. BMC Med 10: 157-160, 2012. https://doi.org/10.1186/1741-7015-10-157

LUBOS E, MESSOW CM, SCHNABEL R, RUPPRECHT HJ, ESPINOLA-KLEIN C, BICKEL C, PEETZ D, POST F, LACKNER KJ, TIRET L, MÜNZEL T, BLANKENBERG S: Resistin, acute coronary syndrome and prognosis results from the AtheroGene study. Atherosclerosis 193: 121-128, 2007. https://doi.org/10.1016/j.atherosclerosis.2006.05.039

MANCIO J, OIKONOMOU EK, ANTONIADES C: Perivascular adipose tissue and coronary atherosclerosis. Heart 104: 1654-1662, 2018. https://doi.org/10.1136/heartjnl-2017-312324

NASHEF SA, ROQUES F, SHARPLES LD, NILSSON J, SMITH C, GOLDSTONE AR, LOCKOWANDT U: EuroSCORE II. Eur J Cardiothorac Surg 41: 734-744, 2012. https://doi.org/10.1093/ejcts/ezs043

NOSALSKI R, GUZIK TJ: Perivascular adipose tissue inflammation in vascular disease. Br J Pharmacol. 174: 3496-3513, 2017. https://doi.org/10.1111/bph.13705

OIKONOMOU EK, MARWAN M, DESAI MY, MANCIO J, ALASHI A, HUTT CENTENO E, THOMAS S, HERDMAN L, KOTANIDIS CP, THOMAS KE, GRIFFIN BP, FLAMM SD, ANTONOPOULOS AS, SHIRODARIA C, SABHARWAL N, DEANFIELD J, NEUBAUER S, HOPEWELL JC, CHANNON KM, ACHENBACH S, ANTONIADES C: Non-invasive detection of coronary inflammation using computed tomography and prediction of residual cardiovascular risk (the CRISP CT study): a post-hoc analysis of prospective outcome data. Lancet 392: 929-939, 2018. https://doi.org/10.1016/S0140-6736(18)31114-0 
PILZ S, WEIHRAUCH G, SEELHORST U, WELLNITZ B, WINKELMANN BR, BOEHM BO, MÄRZ W: Implications of resistin plasma levels in subjects undergoing coronary angiography. Clin Endocrinol (Oxf) 66: 380-386, 2007. https://doi.org/10.1111/j.1365-2265.2007.02743.x

SARACEVIC 1 A, MEDVED I, HRABRIC VLAH S, KOZMAR A, BILIC-ZULLE L, SIMUNDIC AM: The association of systemic inflammatory markers with indicators of stress and cardiac necrosis in patients undergoing aortic valve replacement and revascularization surgeries. Physiol Res 69: 261-274, 2020. https://doi.org/10.33549/physiolres.934243

SPILIOPOULOS K, BAGIATIS V, DEUTSCH O, KEMKES BM, ANTONOPOULOS N, KARANGELIS D, HASCHEMI A: Performance of EuroSCORE II compared to EuroSCORE I in predicting operative and mid-term mortality of patients from a single center after combined coronary artery bypass grafting and aortic valve replacement. Gen Thorac Cardiovasc Sur 62: 103-111. 2014. https://doi.org/10.1007/s11748-013-0311-8

SULLIVAN PG, WALLACH JD, IOANNIDIS JP: Meta-analysis comparing established risk prediction models (EuroSCORE II, STS Score, and ACEF Score) for perioperative mortality during cardiac surgery. Am J Cardiol 118: 1574-1582, 2016. https://doi.org/10.1016/j.amjcard.2016.08.024

VERSTEYLEN MO, TAKX RA, JOOSEN IA, NELEMANS PJ, DAS M, CRIJNS HJ, HOFSTRA L, LEINER T: Epicardial adipose tissue volume as a predictor for coronary artery disease in diabetic, impaired fasting glucose, and non-diabetic patients presenting with chest pain. Eur Heart J Cardiovasc Imaging 13: 517-523, 2012. https://doi.org/10.1093/ehjci/jes024

WANG H, CHEN DY, CAO J, HE ZY, ZHU BP, LONG M: High serum resistin level may be an indicator of the severity of coronary disease in acute coronary syndrome. Chin Med Sci J 24: 161-166, 2009. https://doi.org/10.1016/S1001-9294(09)60082-1 